\title{
Modulatory Effect of Glial-Derived Growth Factor on Addiction
}

\author{
Maryam Jafarian', Mohammadesmaeil Alipour ${ }^{2 *}$ \\ ${ }^{1}$ Shefa Neuroscience Research Center, Khatam Alanbia Hospital, Tehran, Iran
}

${ }^{2}$ School of Advance Technologies in Medicine, Tehran University of Medical Sciences, Tehran, Iran

\section{ABSTRACT}

Introduction: Glial-derived growth factor (GDNF) is an important secretory protein that plays a crutial role in the growth and development of the central and peripheral nervous systems, especially the survival of dopaminergic adult neurons. Several investigations have shown the unique negative modulatoty role of GDNF in drug abuse. Conclusion: This study is a brief description on GDNF and its positive effects as a potential target for the treatment of drug addiction.

\section{Key words:}

1. Glial Cell Line-Derived Neurotrophic Factor

2. Dopaminergic Neurons

3. Central Nervous System

4. Peripheral Nervous System

5. Substance-Related Disorders

*Corresponding Author: Mohammadesmaeil Alipour

E-mail:dralipour@yahoo.com

doi: 10.18869/acadpub.shefa.4.4.116 


\title{
اثر تعديل كننده فاكتور رشد عصبى مشتق از گليا بر اعتياد
}

\author{
مريم جعفريان'، محمد اسماعيل علييور ז"* \\ 'مركز تحقيقات علوم اعصاب شفا، بيمارستان خاتم الانبياء، تهران، ايران \\ rانشكده فناورى هاى نوين بزشكى، دانشكاه علوم يزشكى تهران، تهران، ايران
}

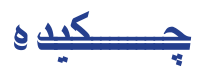

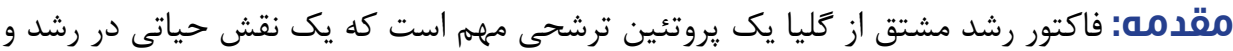

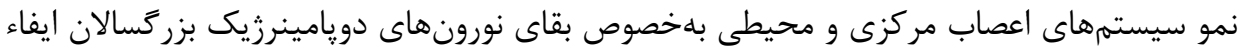

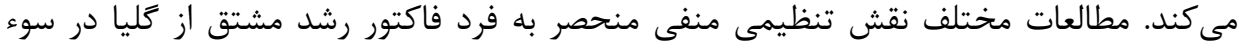

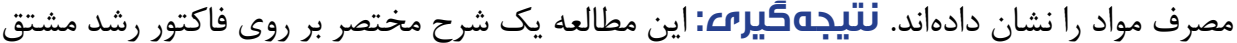
از كليا و اثرات مطلوب آن بهانوان آنوان يك هدف احتمالى براى درمان اعتياد به به مواد است.

| (1. فاكتور رشد عصبى مشتق از

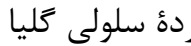

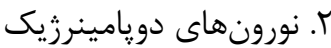

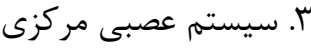

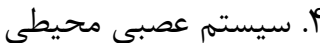
ه. اختلالات وابسته به مصرف مواد 
يروتئينهـاى الحاقـى عصبــى (NCAM) ' نيــز عمــل

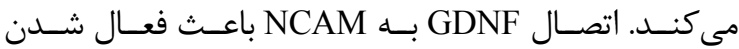

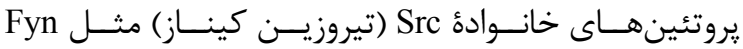

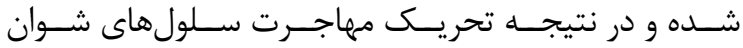

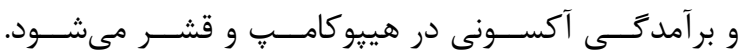

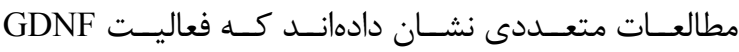

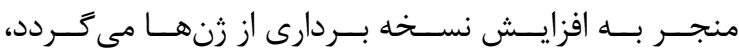

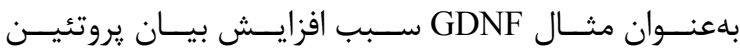

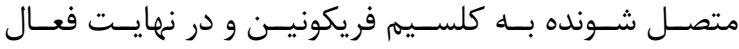

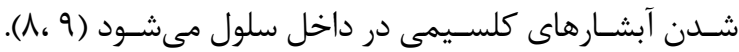

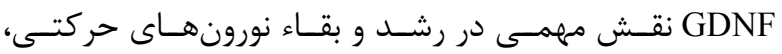

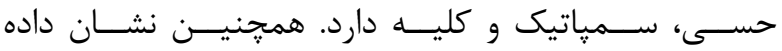

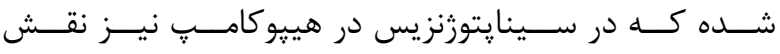

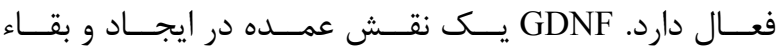

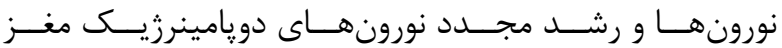

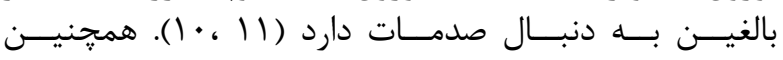

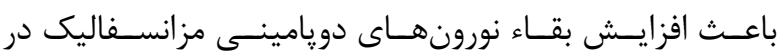

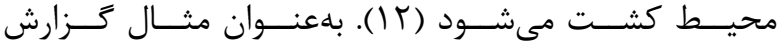

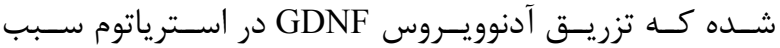

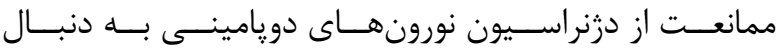

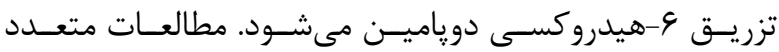

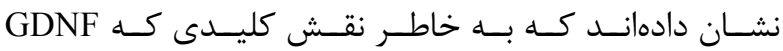

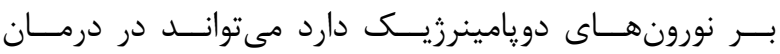

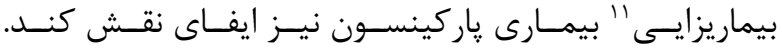

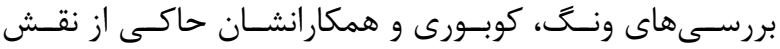

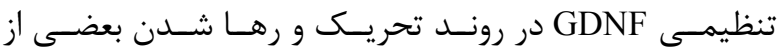

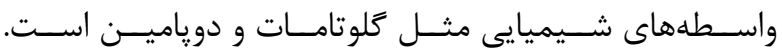

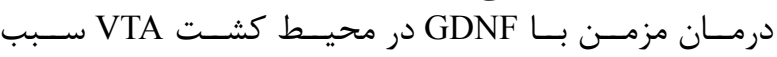

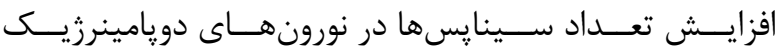

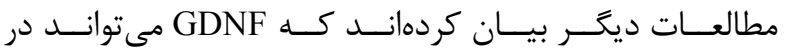

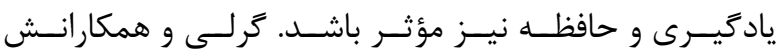

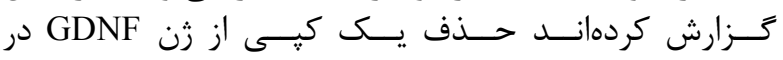

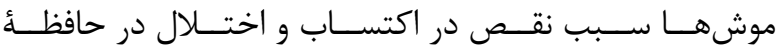

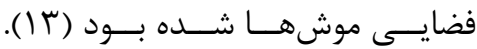

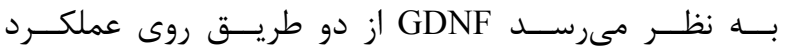

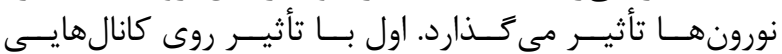

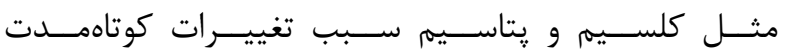

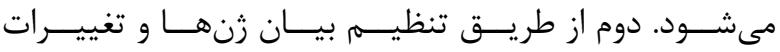

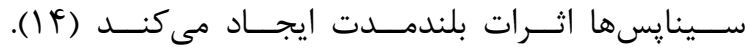

GDNF

كيرندههـاى GDNF، GFR و Ret در ناحيــ VTA

${ }^{1}$ Glial derived neurothrophic factor

${ }^{2}$ Ventral tegmentom area

${ }^{3}$ Nucleuse accombence

${ }^{4}$ Glomerular filtration rate

${ }^{5}$ Rearranged during transfection receptor

${ }^{6}$ Mitogen-activated protein kinase

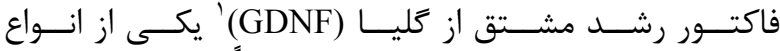

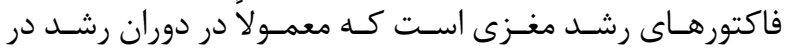

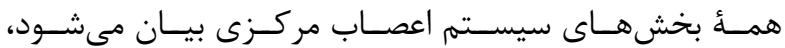

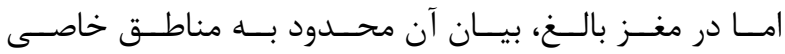

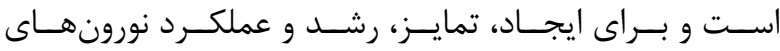

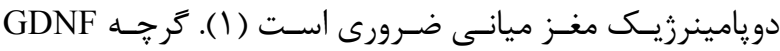

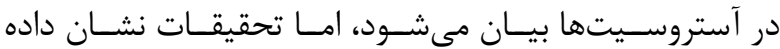

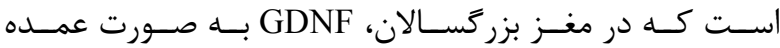

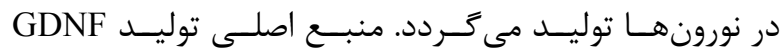

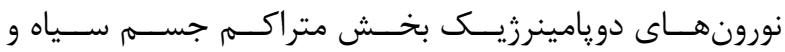

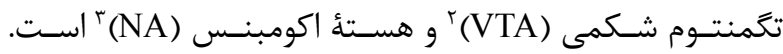

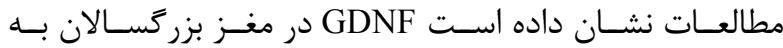

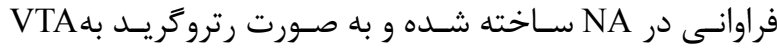

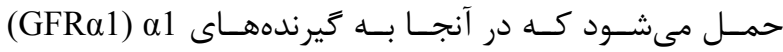

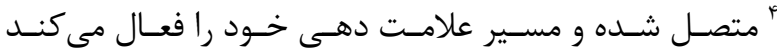

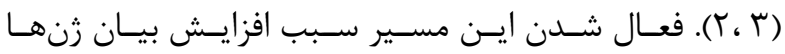

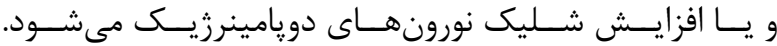

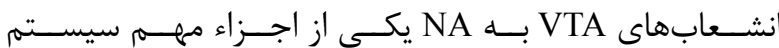

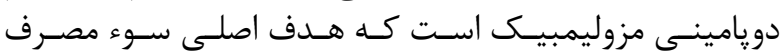

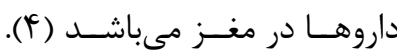
مسير هاى قييامرسانى و عملكرد GDNF

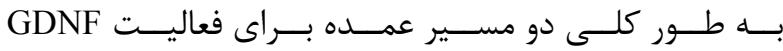

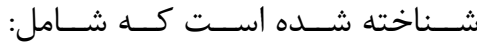

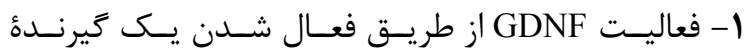

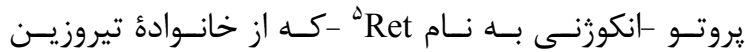

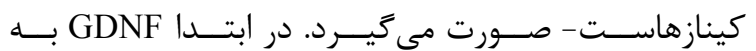

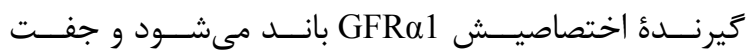

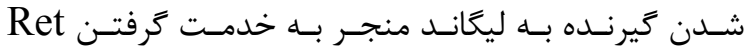

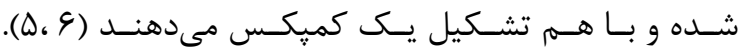

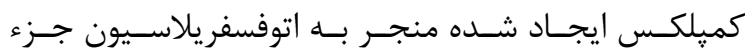

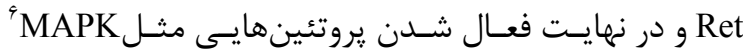

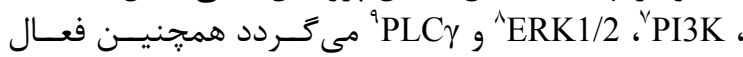

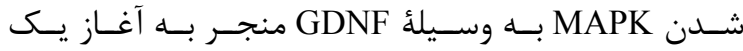

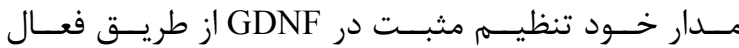

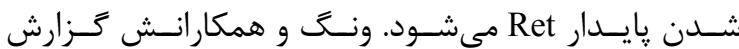

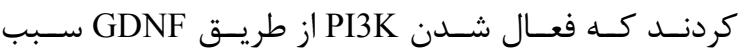

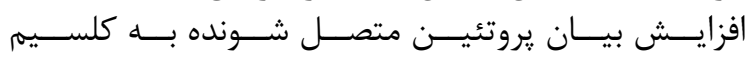

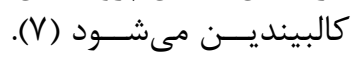

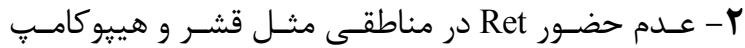

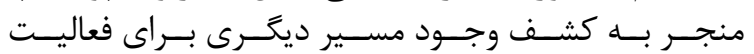

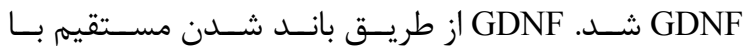

${ }^{7}$ Phoshatidylinositol 3kinase

${ }^{8}$ Extracellular signal-regulated kinase $1 / 2$

${ }^{9}$ Phospholipase $\mathrm{C} \gamma$

${ }^{10}$ Neural cell adhesion molecule

${ }^{11}$ Pathogenicity 


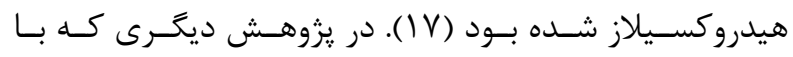

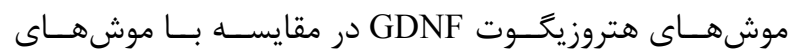

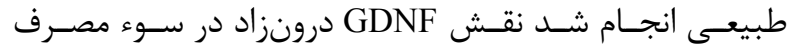

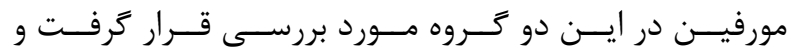

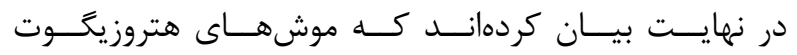

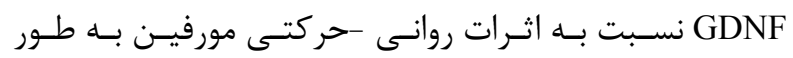

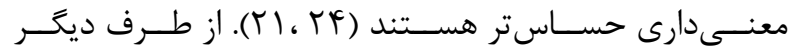

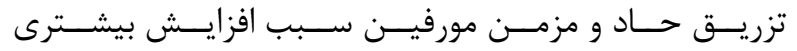

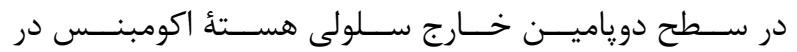

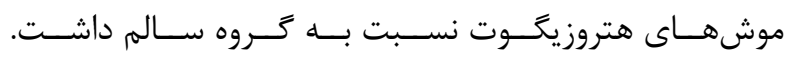

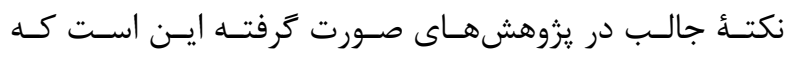

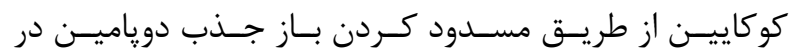

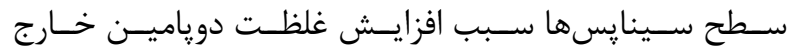

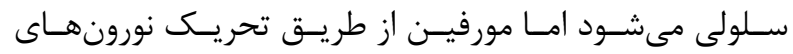

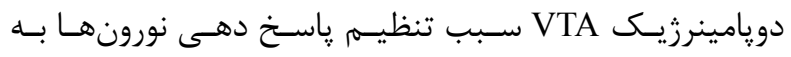

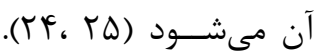

GIDNF

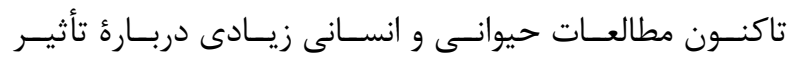

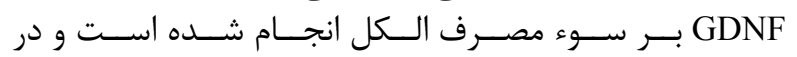

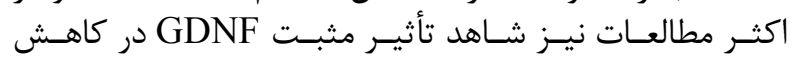

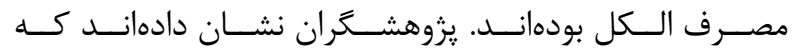

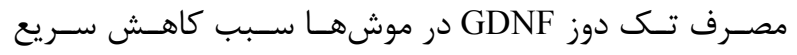

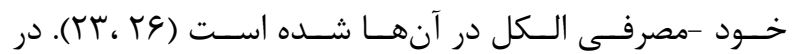

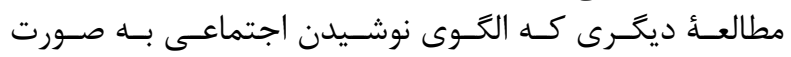

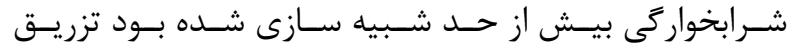

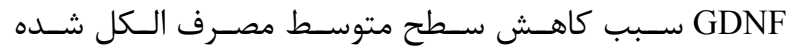

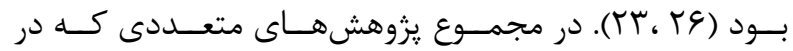

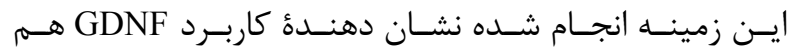

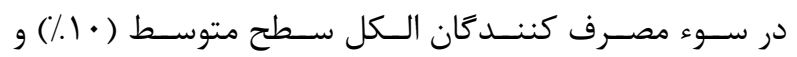

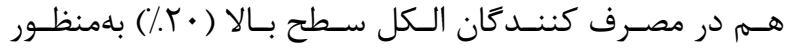

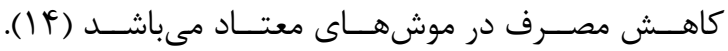

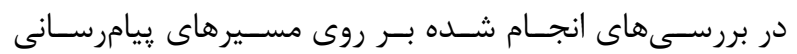

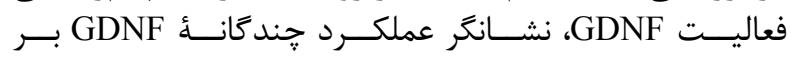

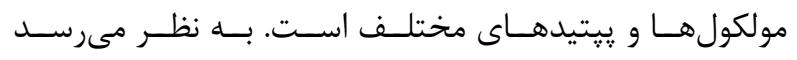

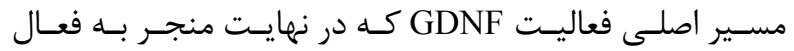

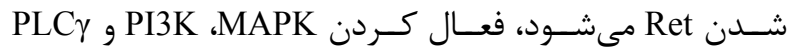

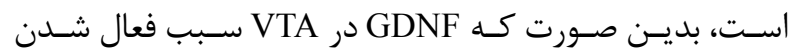

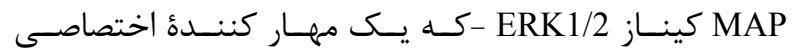

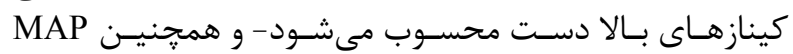

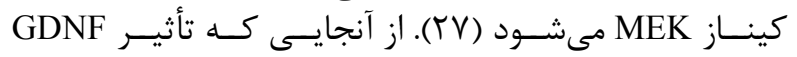

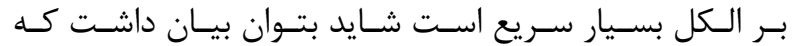

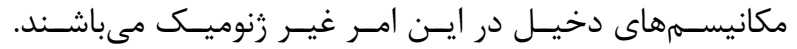

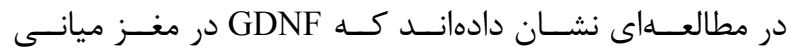

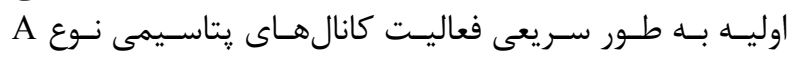

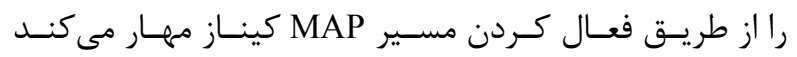

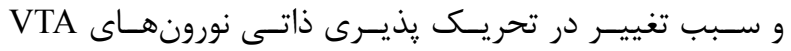

${ }^{12}$ Condition place prifrence

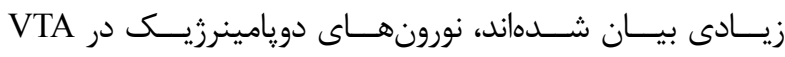

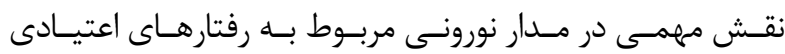

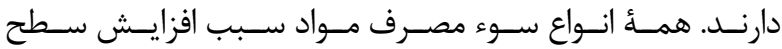

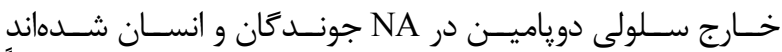

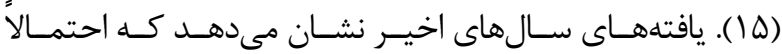

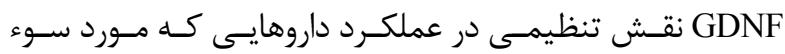

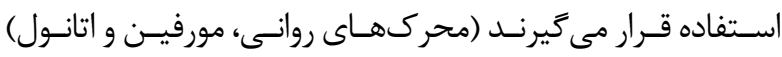

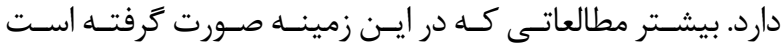

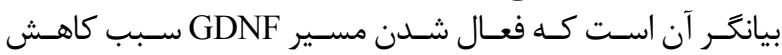

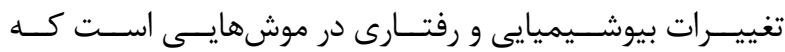

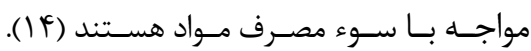
GDNF

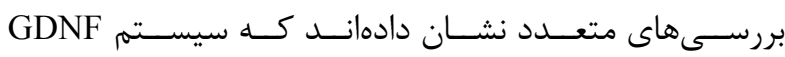

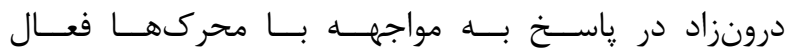

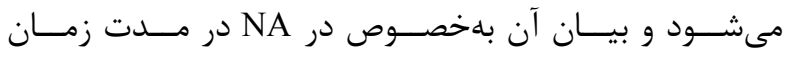

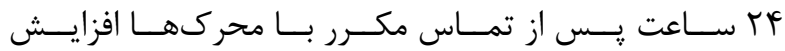

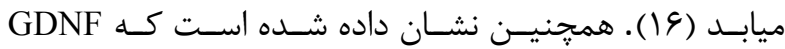

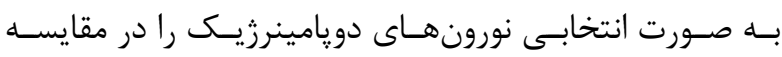

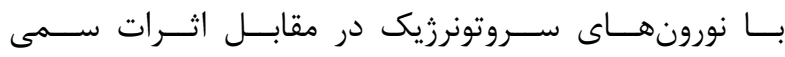

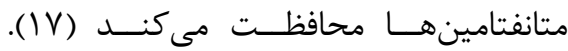

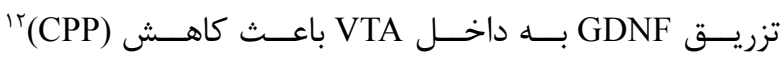

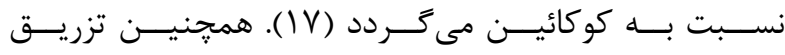

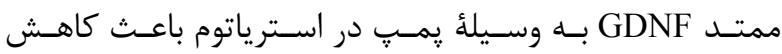

مصـرف كوكائيسن در Rat كرديسـد (19 19 19).

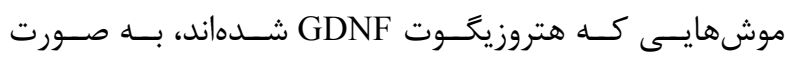

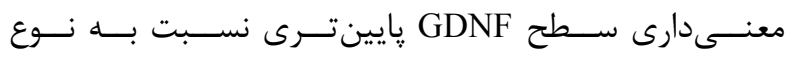

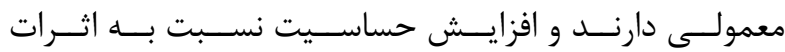

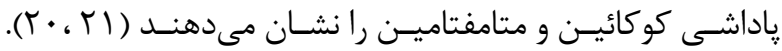

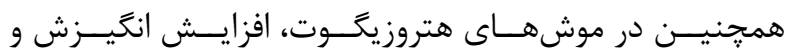

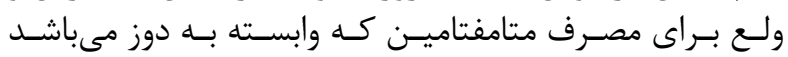

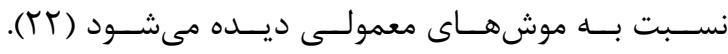

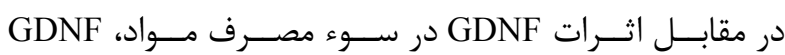

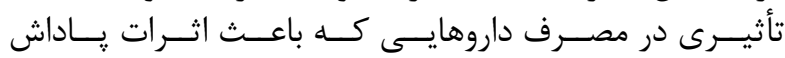

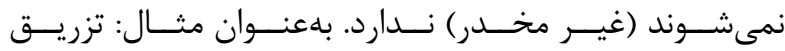

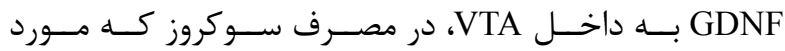

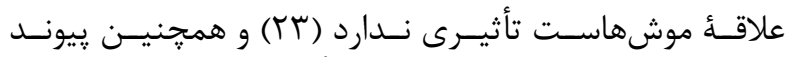

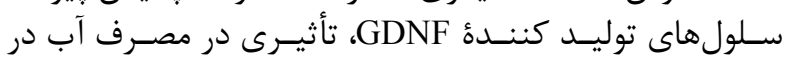

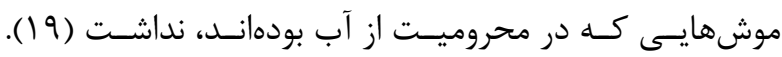

\section{GDNF}

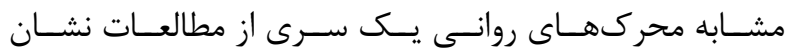

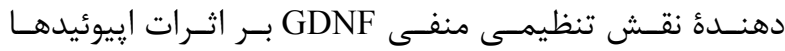

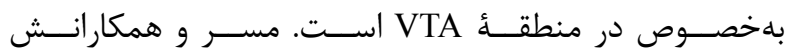

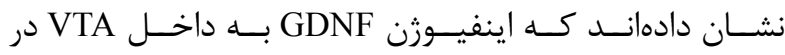

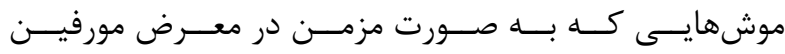

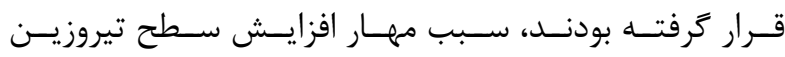




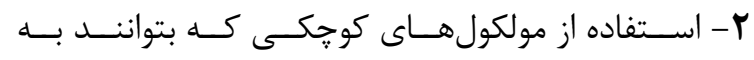

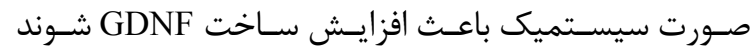

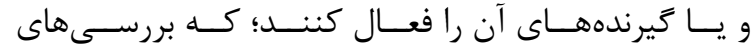

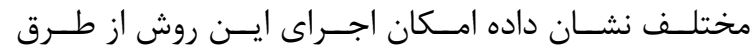

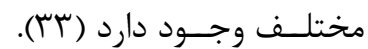

نتيجه كيرى

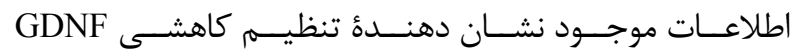

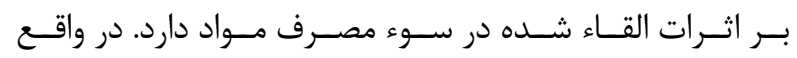

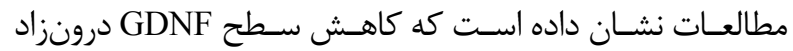

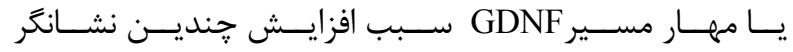

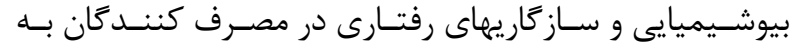

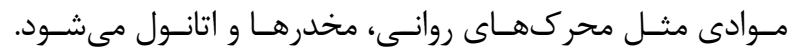

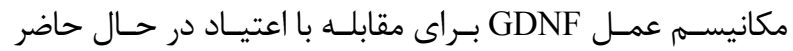

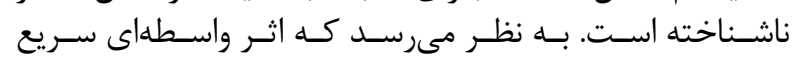

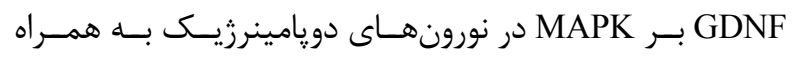

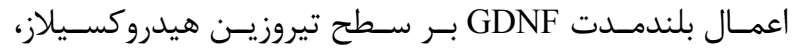

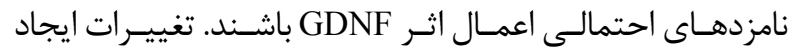

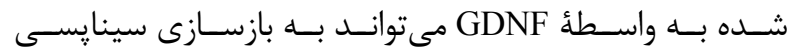

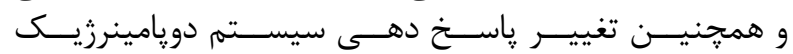

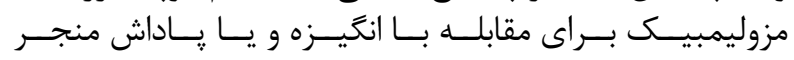

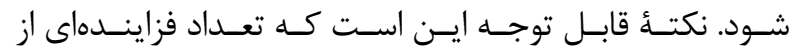

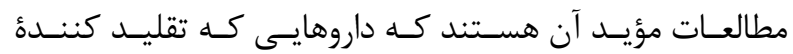

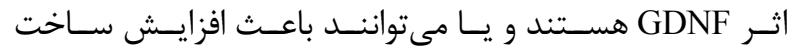

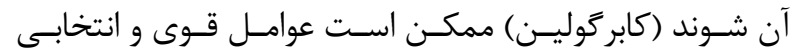

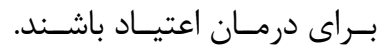

1. Airaksinen MS, Saarma M. The GDNF family: signalling, biological functions and therapeutic value. Nat Rev Neurosci. 2002; 3(5): 383-94.

2. Barroso-Chinea P, Cruz-Muros I, Aymerich MS, Rodríguez-Díaz M, Afonso-Oramas D, Lanciego JL, et al. Striatal expression of GDNF and differential vulnerability of midbrain dopaminergic cells. Eur J Neurosci. 2005; 21(7): 1815-27.

3. Wang J, Carnicella S, Ahmadiantehrani S, He D-Y, Barak S, Kharazia V. Nucleus accumbens-derived glial cell line-derived neurotrophic factor is a retrograde enhancer of dopaminergic tone in the mesocorticolimbic system. J Neurosci. 2010; 30(43): 14502-12.

4. Nestler EJ. Molecular basis of long-term plasticity underlying addiction. Nat Rev Neurosci. 2001; 2(2): 119-28.

5. Jing S, Wen D, Yu Y, Holst PL, Luo Y, Fang M. GDNF-induced activation of the ret protein tyrosine

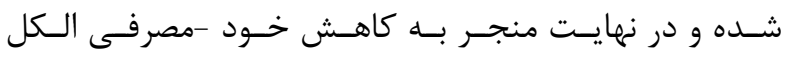

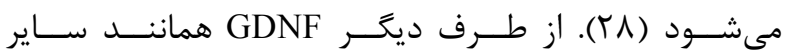

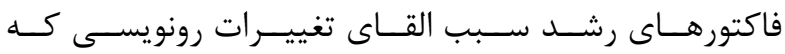

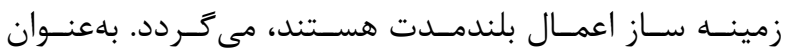

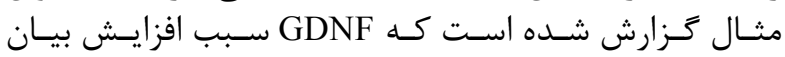

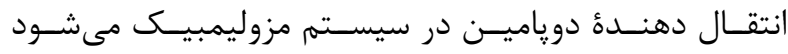

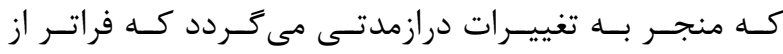

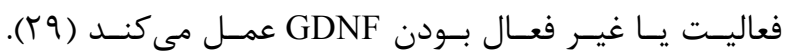

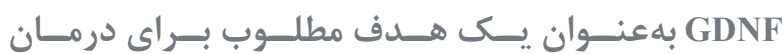
اعتيــاد

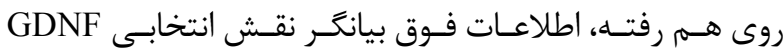

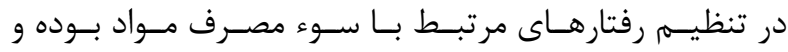

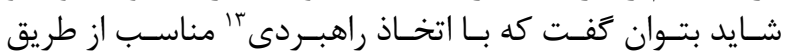

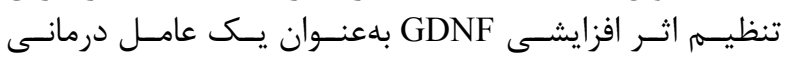

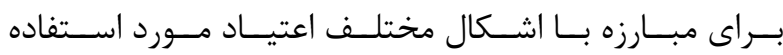

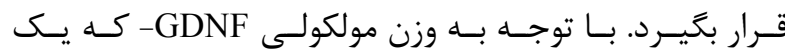

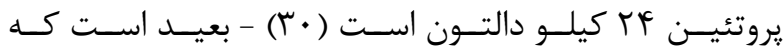

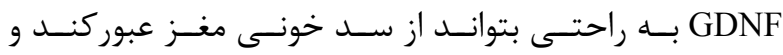

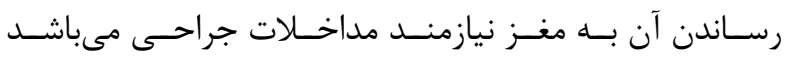

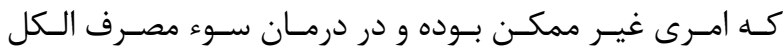

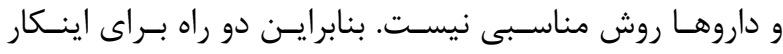

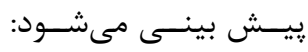

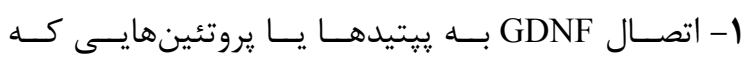

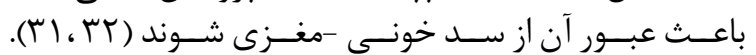

منابع

kinase is mediated by GDNFR- $\alpha$, a novel receptor for GDNF. Cell. 1996; 85(7): 1113-24.

6. Tsui-Pierchala BA, Ahrens RC, Crowder RJ, Milbrandt J, Johnson EM. The long and short isoforms of Ret function as independent signaling complexes. J Biol Chem. 2002; 277(37): 34618-25.

7. Wang H-J, Cao J-P, Yu J-K, Zhang L-C, Jiang Z-J, Gao D-S. Calbindin-D28K expression induced by glial cell line-derived neurotrophic factor in substantia nigra neurons dependent on $\mathrm{PI} 3 \mathrm{~K} / \mathrm{Akt} / \mathrm{NF}-\kappa \mathrm{B}$ signaling pathway. Euro J Pharmacol. 2008; 595(1-3): 7-12.

8. Paratcha G, Ledda F, Ibáñez CF. The neural cell adhesion molecule NCAM is an alternative signaling receptor for GDNF family ligands. Cell. 2003; 113(7): 867-79.

9. Chao CC, Ma YL, Chu KY, Lee EH. Integrin $\alpha$ v and NCAM mediate the effects of GDNF on DA neuron survival, outgrowth, DA turnover and motor activity in 
rats. Neurobiol Aging. 2003; 24(1): 105-16.

10. Beck KD, Valverde J, Alexi T, Poulsen K, Moffat B, Vandlen RA. Mesencephalic dopaminergic neurons protected by GDNF from axotomy-induced degeneration in the adult brain. Nature. 1995; 373(1): 339-41.

11. Pascual A, Hidalgo-Figueroa M, Piruat JI, Pintado CO, Gómez-Díaz R, López-Barneo J. Absolute requirement of GDNF for adult catecholaminergic neuron survival. Nat Neurosci. 2008; 11(7): 755-61.

12. Lin L-FH, Doherty DH, Lile JD, Bektesh S, Collins F. GDNF: a glial cell line-derived neurotrophic factor for midbrain dopaminergic neurons. Sciences. 1993; 260: 1130 .

13. Gerlai R, McNamara A, Choi-Lundberg D, Armanini M, Ross J, Powell-Braxton L. Impaired water maze learning performance without altered dopaminergic function in mice heterozygous for the GDNF mutation. Euro J Neurosci. 2001; 14(7): 1153-63.

14. Carnicella S, Ron D. GDNF-a potential target to treat addiction. Pharmacol Therapeut. 2009; 122(1):9-18.

15. Volkow ND, Fowler JS, Wang G-J, Swanson JM, Telang F. Dopamine in drug abuse and addiction: results of imaging studies and treatment implications. Arch Neurol-Chicago. 2004; 9: 557-69.

16. Semba Ji, Akanuma N, Wakuta M, Tanaka N, Suhara T. Alterations in the expressions of mRNA for GDNF and its receptors in the ventral midbrain of rats exposed to subchronic phencyclidine. Mol Brain Res. 2004; 124(1): 88-95.

17. Messer CJ, Eisch AJ, Carlezon WA, Whisler K, Shen L, Wolf DH1. Role for GDNF in biochemical and behavioral adaptations to drugs of abuse. Neuron. 2000; 26(1): 247-57.

18. Green-Sadan T, Kuttner Y, Lublin-Tennenbaum T, Kinor N, Boguslavsky Y, Margel S. Glial cell linederived neurotrophic factor-conjugated nanoparticles suppress acquisition of cocaine self-administration in rats. Exp Neurol. 2005; 194(1): 97-105.

19. Green-Sadan T, Kinor N, Roth-Deri I, GeffenAricha R, Schindler CJ, Yadid G. Transplantation of glial cell line-derived neurotrophic factor-expressing cells into the striatum and nucleus accumbens attenuates acquisition of cocaine self-administration in rats. Euro J Neurosci. 2003; 18(7): 2093-8.

20. Airavaara M, Planken A, Gäddnäs H, Piepponen TP, Saarma M, Ahtee L. Increased extracellular dopamine concentrations and FosB $/ \Delta$ FosB expression in striatal brain areas of heterozygous GDNF knockout mice. Euro J Neurosci. 2004; 20(9): 2336-44.

21. Niwa M, Nitta A, Yamada Y, Nakajima A, Saito K, Seishima M. An inducer for glial cell line-derived neurotrophic factor and tumor necrosis factor- $\alpha$ protects against methamphetamine-induced rewarding effects and sensitization. Biol Psychiat. 2007; 61(7): 890-901.

22. Yan Y, Yamada K, Niwa M, Nagai T, Nitta A, Nabeshima T. Enduring vulnerability to reinstatement of methamphetamine-seeking behavior in glial cell linederived neurotrophic factor mutant mice. Faseb J. 2007; 21(9): 1994-2004.

23. Carnicella S, Kharazia V, Jeanblanc J, Janak PH, Ron D. GDNF is a fast-acting potent inhibitor of alcohol consumption and relapse. Proceedings of the National Academy of Sciences. 2008; 105(23): 8114-9.

24. Airavaara M, Tuomainen H, Piepponen T, Saarma M, Ahtee L. Effects of repeated morphine on locomotion, place preference and dopamine in heterozygous glial cell line-derived neurotrophic factor knockout mice. Genes, Brain Behav. 2007; 6(3): 287-98.

25. Airavaara M, Mijatovic J, Vihavainen T, Piepponen TP, Saarma M, Ahtee L. In heterozygous GDNF knockout mice the response of striatal dopaminergic system to acute morphine is altered. Synapse. 2006; 59(6): 321-9.

26. He D-Y, McGough NN, Ravindranathan A, Jeanblanc J, Logrip ML, Phamluong K, et al. Glial cell line-derived neurotrophic factor mediates the desirable actions of the anti-addiction drug ibogaine against alcohol consumption. J Neurosci. 2005; 25(3): 619-28.

27. Sweatt JD. The neuronal MAP kinase cascade: a biochemical signal integration system subserving synaptic plasticity and memory. J Neurochem. 2001; 76(1): 1-10.

28. Yang F, Feng L, Zheng F, Johnson SW, Du J, Shen L. GDNF acutely modulates excitability and A-type $\mathrm{K}+$ channels in midbrain dopaminergic neurons. Nat Neurisci. 2001; 4(11): 1071-8.

29. Consales C, Volpicelli F, Greco D, Leone L, ColucciD'Amato L, Perrone-Capano C. GDNF signaling in embryonic midbrain neurons in vitro. Brain Res. 2007; 1159: 28-39.

30. Kastin AJ, Akerstrom V, Pan W. Glial cell line derived neurotrophic factor does not enter normal mouse 
brain. Neurosci Lett. 2003; 340(3): 239-41.

31. Kilic Ü, Kilic E, Dietz GP, Bähr M. Intravenous TAT-GDNF is protective after focal cerebral ischemia in mice. Stroke. 2003; 34(5): 1304-10.

32. Boado RJ, Zhang Y, Zhang Y, Wang Y, Pardridge
WM. GDNF fusion protein for targeted-drug delivery across the human blood-brain barrier. Biotechnol Bioeng. 2008; 100(2): 387-96.

33. Shoptaw S, Heinzerling KG, Rotheram-Fuller E, Steward T, Wang J, Swanson A-N. Drug Alcohol Depen. 2008; 96(1): 222-32. 